\title{
DETC2001/VIB-21616
}

\section{COMPARISON OF THE DYNAMICS OF LOW IMMERSION MILLING AND CUTTING WITH VARYING SPINDLE SPEED}

\author{
Tamás Insperger \\ Department of Applied Mechanics \\ Budapest University of Technology and Economics \\ Budapest, $\mathrm{H}-1521$, Hungary \\ e-mail: inspi@mm.bme.hu \\ Sri N. Namachchivaya \\ Gábor Stépan
Department of Applied Mechanics \\ Budapest University of Technology and Economics \\ Budapest, $\mathrm{H}-1521$, Hungary \\ e-mail: stepan@mm.bme.hu
Department of Aeronautical and Astronautical Engineering
University of Illinois at Urbana-Champaign
306 Talbot Lab, 104 South Wright Str, Urbana, IL 61801
E-mail: sri@nsgsun.aae.uiuc.edu

\begin{abstract}
The stability properties of cutting processes are strongly limited by the so-called regenerative effect. This effect is originated in the presence of a time-delay in the dynamical system of the machine tool. This delay is inversely proportional to the cutting speed. Consequently, conventional cutting with a single-edge tool is modeled by an autonomous delay-differential equation (DDE). In case of milling, the varying number of cutting edges results in a kind of parametric excitation, and the corresponding mathematical model is a non-autonomous DDE. In case of low-immersion milling, this affects the stability boundaries in a substantial way. Cutting with varying spindle speed results non-autonomous DDEs where the time delay itself depends on the time periodically. A new semi-discretization method is proposed to handle the stability of these non-autonomous systems. The stability properties and corresponding bifurcations are compared in the above different cases of machining.
\end{abstract}

\section{INTRODUCTION}

The so-called regenerative effect often results self-excited vibrations on machine tools. The tool cuts a wavy surface formed in the previous cut. This surface was formed by the tool edge during the previous round of the workpiece. Since the actual cutting force acting on the machine tool depends on the actual chip thickness, the variation of the cutting force depends on the difference of the present and the past positions of the tool. The past position of the tool means the position $x$ at $t-\tau$, where $t$ is the actual time, and $\tau$ is the time of one revolution of the workpiece. This explains why the time delay $\tau$ is inversely proportional to the cutting speed.

It is a rule of thumb that delay tends to destabilize any dynamical system. The so-called stability charts represent those domains in the parameter plane where stable stationary cutting exists. Traditionally, those parameters are represented in these charts, which can be influenced via the chosen technology, i.e. via the choice of the spindle speed, depth of cut and feed rate, or in another coordinate system, via the cutting speed, chip width and chip thickness. The conventional stability charts for turning show a complicated structure. The so-called lobes in these stability charts are well known since the early sixties (see Tlusty et al., 1962, Tobias, 1965). The charts can be calculated by analytical methods based on the analysis of the transcendental characteristic functions of the corresponding autonomous DDEs (see, for example, Stépán, 1989,1998).

In case of milling, the time delay is shorter, it is equal to the tooth pass period, that is to the time of one tool revolution divided by the number $z$ of the teeth on the milling tool. The major change in the mechanical model is, however, that timedependent periodic parameters appear in the system. This parametric excitation is due to the time varying number of the active teeth of the milling tool, and/or to the periodic change of the cutting force components of each tooth in the directions of the vibration modes of the machine tool.

This parametric excitation does not cause major changes in the stability charts in case of low spindle speeds and active teeth number greater than 3 . This means that the time 
averaging methods leading to approximate autonomous DDEs serve equally satisfactory results as the numerical simulation, experiments, or other techniques (see, e.g., Tobias, 1965, Minis and Yanushevsky, 1993). For high-speed milling, and/or low immersion milling, new stability and bifurcation phenomena occur, which cannot be identified by the conventional analytical methods. Numerical simulation of Balachandran and Zhao (2000), special approximate analytical methods of Budak and Altintas (1995), Davies et al. (2000), Corpus and Endres (2000), Seagalman and Butcher (2000) lead to the realization of these new stability properties. The method of Insperger and Stépán (2000) based on a transformation proposed by Fargue (1973) also explored the connection of the conventional stability charts of turning and the new ones of milling.

The periodically varying cutting speed results parametric excitation in the time delay. Some experts (like Sexton and Stone, 1978) predicted improvements in stability properties by a factor of 10 for properly chosen parameter values. The great expectations were partly based on the famous problem of stabilizing inverted pendulums by parametric excitation (see, for example, Insperger and Horváth, 2000). In spite of some reports on successful experiments, the stability investigations of cutting with time varying spindle speeds were not reliable enough to present a breakthrough in this field. With the help of a new method described in this paper, the similarities and differences in the stability properties of low immersion milling and time varying cutting are explored. The stability charts of turning, milling and time varying cutting are derived with a unified approach, and the existing methods and models are also compared in the subsequent sections.

\section{BASIC MODEL FOR TURNING}

Figure 1 shows the simplest one degree of freedom mechanical model of turning in case of orthogonal cutting. The corresponding equation of motion of the turning process reads

$$
\ddot{x}(t)+2 \zeta \omega_{n} \dot{x}(t)+\omega_{n}{ }^{2} x(t)=\frac{k_{1}}{m}(x(t-\tau)-x(t)),
$$

where $\zeta$ is the relative damping factor, $\omega_{n}$ is the (lowest) natural angular frequency of the machine tool, $m$ is the modal mass and $\tau=2 \pi / \Omega$ is the time delay, where $\Omega$ is the spindle speed of the tool expressed as angular velocity with unit $[1 / \mathrm{s}]$. The linearized cutting force variation is expressed by means of the cutting force coefficient $k_{1}$, which is the derivative of the cutting force with respect to the chip thickness at its desired stationary value. Introduce the dimensionless time $\tilde{t}=\omega_{n} t$, and by abuse of notation, drop the tilde immediately. Then the equation of motion assumes the form

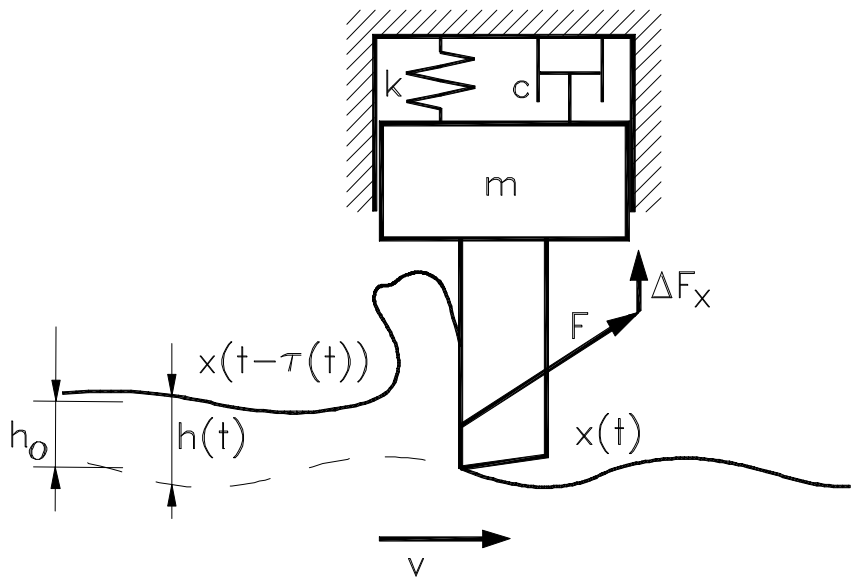

Fig. 1. Regenerative mechanical model of cutting

$$
\ddot{x}(t)+2 \zeta \dot{x}(t)+\left(1+\frac{k_{1}}{m \omega_{n}{ }^{2}}\right) x(t)=\frac{k_{1}}{m \omega_{n}{ }^{2}} x\left(t-\omega_{n} \tau\right)
$$

In the following, the stability charts will be shown in the plane of the dimensionless cutting force coefficient $k_{1} /\left(m \omega_{n}{ }^{2}\right)$ and the dimensionless spindle speed $\Omega / \omega_{n}$. The dimensionless cutting coefficient is the ratio of the cutting coefficient and the modal stiffness, and it is linearly proportional to the chip width, while the dimensionless spindle speed is linearly proportional to the cutting speed.

\section{MODELS OF TIME VARYING CUTTING}

As explained in the Introduction, both the milling process and the turning with varying spindle speed are non-stationary cutting processes. The corresponding mathematical models are similar in the sense that the equations of motion are nonautonomous, time-periodic DDEs.

The dimensionless equation of motion of milling reads

$$
\ddot{x}(t)+2 \zeta \dot{x}(t)+\left(1+\frac{k_{1 p}(t)}{m \omega_{n}{ }^{2}}\right) x(t)=\frac{k_{1 p}(t)}{m \omega_{n}{ }^{2}} x\left(t-\omega_{n} \tau\right),
$$

where $k_{1 p}(t)$ is $\tau=2 \pi /(z \Omega)$ periodic according to the tooth pass excitation effect with $z$ standing for the number of teeth. In this case, the coefficients of the actual and delayed state variables are periodic, and this periodicity is just equal to the time delay. This time-periodicity of the cutting coefficient is often 'almost' piece-wise constant due to the sudden changes of the actual number of active teeth (see Fig. 2).

The equation of turning with varying spindle speed reads

$$
\ddot{x}(t)+2 \zeta \dot{x}(t)+\left(1+\frac{k_{1}}{m \omega_{n}{ }^{2}}\right) x(t)=\frac{k_{1}}{m \omega_{n}{ }^{2}} x\left(t-\omega_{n} \tau(t)\right),
$$




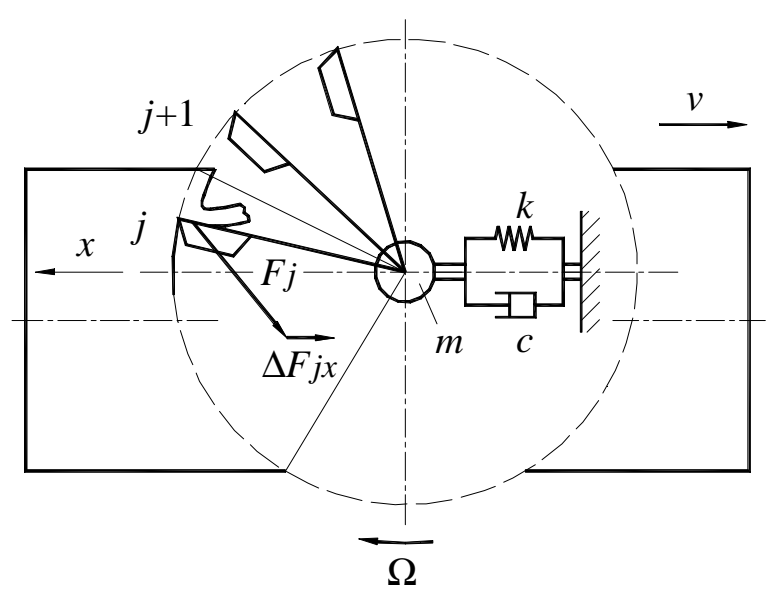

Fig. 2. Mechanical model of the milling process

where the time delay itself varies periodically with period $T$, and in general, $T \neq \tau_{0}$, since the time period in the change of the cutting speed is usually larger than the average time delay $\tau_{0}=\int_{0}^{T} \tau(t) \mathrm{d} t / T$. The coefficients are constant values, but in the sense of the theory of functional differential equations, this equation is also non-autonomous (Hale and Lunel, 1993).

One possible approach to this problem is to transform this equation to a DDE with periodic coefficients using a kind of power series of the delayed term. Consider the time delay in the form $\tau(t)=\tau_{0}-\tau_{1} p(t)$ with a sufficiently small delay variation amplitude $\tau_{1}$, and $p(t)=p(t+T)$ is periodic. Then the first order Taylor series approximation with respect to $\tau_{1}$ assumes the form

$$
x\left(t-\tau_{0}+\tau_{1} p(t)\right) \approx x\left(t-\tau_{0}\right)+\dot{x}\left(t-\tau_{0}\right) \tau_{1} p(t) .
$$

The substitution of this approximation into (4) results a DDE with a constant time delay $\tau_{0}$ only:

$$
\begin{aligned}
\ddot{x}(t)+ & 2 \zeta \dot{x}(t)+\left(1+\frac{k_{1}}{m \omega_{n}{ }^{2}}\right) x(t)= \\
& \frac{k_{1}}{m \omega_{n}{ }^{2}} x\left(t-\omega_{n} \tau_{0}\right)+\frac{k_{1}}{m \omega_{n}{ }^{2}} \tau_{1} p(t) \dot{x}\left(t-\omega_{n} \tau_{0}\right),
\end{aligned}
$$

while the first derivative of the delayed term also appears with a time-periodic coefficient.

The infinite dimensional version of the Floquet Theory developed for functional differential equations (see Farkas, 1994) provides the theoretical basis for the stability analysis of these equations. The linear systems are asymptotically stable in Lyapunov sense if and only if the characteristic multipliers $\mu$ of the so-called monodromy operator are located in the open unit disc of the complex plane. The nature of the bifurcation at the boundary of stability is relevant in practice, since it determines the frequencies of the arising vibrations. Three types of bifurcations are possible when a relevant characteristic multiplier $\left|\mu_{1}\right|=1$ just crosses the unit disc:

1. $\mu_{1}=\bar{\mu}_{2}$, this is topologically equivalent to the Hopf bifurcation of autonomous systems, often called secondary Hopf bifurcation.

2. $\mu_{1}=1$, this is topologically equivalent to the saddlenode bifurcation of autonomous systems.

3. $\mu_{1}=-1$, this type of bifurcation is called flip or period doubling bifurcation. There is no topologically equivalent type of bifurcation for autonomous systems.

It can be shown that only the Hopf bifurcation may occur in the special autonomous case of Eq. (2). For milling, period doubling is also possible (see Corpus and Endres, 2000), however, $\mu_{1}=1$ cannot occur in the case of the corresponding Eq. (3). Substitution of $x\left(t+\omega_{n} \tau\right)=\mu_{1} x(t)=x(t)$ yields zero in the right hand side, and the remaining left hand side is always stable because of the positive damping and stiffness. In the case of turning with time varying cutting speed, Eq. (4) and (6) may present all the three types of bifurcations.

In the following, we will determine the stability charts of Eq. (3), (6) and (4).

\section{SEMI-DISCRETIZATION FOR DDE MODELS WITH TIME PERIODIC COEFFICIENTS}

By semi-discretization we mean the partial discretization of the DDE at the time delay only. The basic idea of this method is presented in this section. Consider the second order $T$-periodic DDE:

$$
\ddot{x}(t)+b_{0}(t) \dot{x}(t)+c_{0}(t) x(t)=b_{1}(t) \dot{x}\left(t-\tau_{0}\right)+c_{1}(t) x\left(t-\tau_{0}\right),
$$

that is a generalization of the mathematical models (3) and (6) with constant time delays.

Divide the time period $T$ into $k$ intervals of length $\Delta t=\tau_{0} /(n+1 / 2)$, where the integer $n$ is called as approximation number. If the principal period $T$ is not a multiple of the interval length $\Delta t$, then an approximate principal period should be applied: $\tilde{T}=k \Delta t$. By decreasing $\Delta t$, that is, by increasing the approximation number $n$, the error decreases.

In the time interval $t \in\left[t_{i}, t_{i+1}\right], i=0,1, \ldots, k-1$ with length $t_{i+1}-t_{i}=\Delta t$, Eq. (7) can be approximated with the following, second order, autonomous ordinary differential equation

$$
\ddot{x}(t)+b_{0 i} \dot{x}(t)+c_{0 i} x(t)=f_{i-n},
$$

where the constant excitation on the right side is 


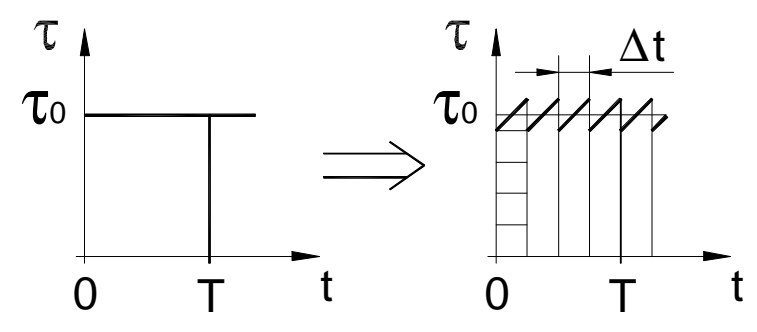

Fig. 3. Discretized time delay

$$
f_{i-n}=b_{1 i} \dot{x}_{i-n}+c_{1 i} x_{i-n} \approx b_{1 i} \dot{x}\left(t-\tau_{0}\right)+c_{1 i} x\left(t-\tau_{0}\right),
$$

and the coefficients $b_{0 i}, c_{0 i}, b_{1 i}$ and $c_{1 i}$ are the constant approximations (say, average values) of the time dependent coefficients $b_{0}(t), c_{0}(t), b_{1}(t)$ and $c_{1}(t)$ in the $i^{\text {th }}$ interval, respectively. This discretization corresponds to a piecewise linear approximation of the constant time delay, as shown in Fig. 3.

Eq. (8) can be solved in closed form for each time interval $\left[t_{i}, t_{i+1}\right]$. With this solution, the system state can be calculated at the time $t_{i+1}$ as a function of the state variables at the time $t_{i}$ which serve as initial conditions for (8). As explained in details by Insperger and Stépán (2001), the connection of these states is given by the discrete map

$$
\mathbf{y}_{i+1}=\mathbf{A}_{i} \mathbf{y}_{i},
$$

where

$$
\begin{gathered}
\mathbf{y}_{i}=\operatorname{col}\left(\dot{x}_{i} x_{i} f_{i} \cdots f_{i-n}\right), \\
\mathbf{A}_{i}=\left(\begin{array}{ccccccc}
A_{1 i} & A_{2 i} & 0 & 0 & \cdots & 0 & A_{3 i} \\
A_{4 i} & A_{5 i} & 0 & 0 & \cdots & 0 & A_{6 i} \\
b_{1 i} & c_{1 i} & 0 & 0 & \cdots & 0 & 0 \\
0 & 0 & 1 & 0 & \cdots & 0 & 0 \\
\vdots & \vdots & \vdots & \ddots & \ddots & \vdots & \vdots \\
0 & 0 & 0 & 0 & \ddots & 0 & 0 \\
0 & 0 & 0 & 0 & \cdots & 1 & 0
\end{array}\right),
\end{gathered}
$$

with

$$
\begin{gathered}
A_{1 i}=\frac{1}{\omega_{i}}\left(\gamma_{i} \sin \left(\omega_{i} \Delta t\right)+\omega_{i} \cos \left(\omega_{i} \Delta t\right)\right) \exp \left(\gamma_{i} \Delta t\right), \\
A_{2 i}=-\frac{\gamma_{i}^{2}+\omega_{i}^{2}}{\omega_{i}} \sin \left(\omega_{i} \Delta t\right) \exp \left(\gamma_{i} \Delta t\right), \\
A_{3 i}=-\frac{1}{c_{0 i}} A_{2 i}
\end{gathered}
$$

$$
\begin{gathered}
A_{4 i}=\frac{1}{\omega_{i}} \sin \left(\omega_{i} \Delta t\right) \exp \left(\gamma_{i} \Delta t\right), \\
A_{5 i}=\left(\cos \left(\omega_{i} \Delta t\right)-\frac{\gamma_{i}}{\omega_{i}} \sin \left(\omega_{i} \Delta t\right)\right) \exp \left(\gamma_{i} \Delta t\right), \\
A_{6 i}=\frac{1}{c_{0 i}}\left(1-A_{5 i}\right)
\end{gathered}
$$

and

$$
\begin{gathered}
\gamma_{i}=-\frac{b_{0 i}}{2}, \\
\omega_{i}=\frac{1}{2} \sqrt{4 c_{0 i}-b_{0 i}{ }^{2}} .
\end{gathered}
$$

The transition matrix $\Phi$ makes connection between the states of the system at time $t_{0}$ and $t_{k}=t_{0}+T$, which is, actually, a kind of finite dimensional approximation of the monodromy operator.

The principal period is - exactly or approximately - a $k$ multiple of the interval length $\Delta t$. Thus, the transition matrix can be given by coupling the solutions for each interval. This results

$$
\Phi=\mathbf{A}_{k-1} \mathbf{A}_{k-2} \ldots \mathbf{A}_{1} \mathbf{A}_{0}
$$

The criterion of asymptotic stability is that all eigenvalues $\mu$ of the transition matrix $\Phi$ are in modulus less than one.

Equations (3) and (6) can be analyzed according to the presented method, since they are special cases of Eq. (7). However, the analysis of Eq. (4) requires further extension of the semi-discretization method for time-periodic delays.

\section{SEMI-DISCRETIZATION FOR DDE MODELS WITH TIME-VARYING DELAY}

If the time delay varies as shown by Eq. (4), the discretization method should be modified. Suppose the simple case, when the variation is piecewise constant

$$
\tau(t)=\left\{\begin{array}{llc}
\tau_{0}+\tau_{1} & \text { if } & 0<t \leq T / 2 \\
\tau_{0}-\tau_{1} & \text { if } & T / 2<t \leq T
\end{array} .\right.
$$

In this case, the same discretization method can be used, with different approximation parameters for the different time delay values. For $0<t \leq T / 2$, the approximation parameter is $n$, while for $T / 2<t \leq T$, it is $m$ (see Fig. 4). This requires the following conditions to be satisfied:

$$
\tau_{0}+\tau_{1}=(n+1 / 2) \Delta t, \quad \tau_{0}-\tau_{1}=(m+1 / 2) \Delta t,
$$




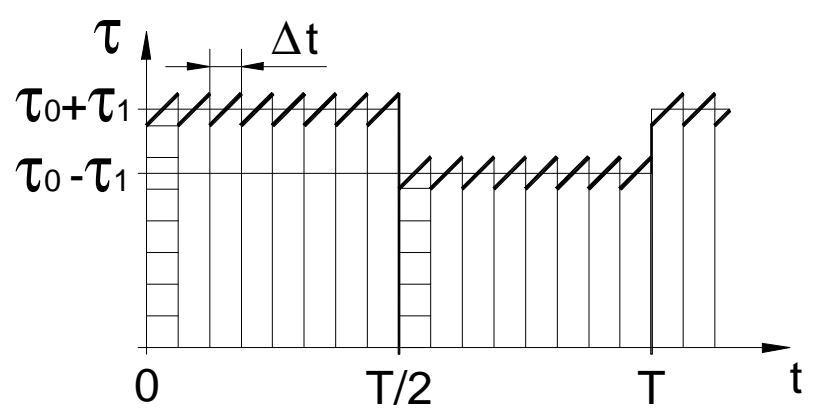

Fig. 4. Discretization of varying time delay

where $n>m$, of course.. For $t \in[0, T / 2)$, the same discrete map is to be used as Eq. (12). For the case $t \in[T / 2, T)$, the discrete map has a form similar to Eq. (12), but the elements $A_{i 3}$ and $A_{i 6}$ are moved form positions $(1, n+2)$ and $(2, n+2)$ to the positions $(1, m+2)$ and $(2, m+2)$, respectively. Then, the transition matrix $\Phi$ can be determined according to Eq. (21).

\section{STABILITY CHARTS}

Stability charts can be created through point by point investigation of the parameter plane $z \Omega / \omega_{n}-k_{1} /\left(m \omega_{n}{ }^{2}\right)$, where $z$ is the number of tool teeth, which is 1 for turning. The boundary curves are given by refining the resolution at the stability boundaries.

In case of Eq. (3), the time varying effect was considered with the following piece-wise constant function

$$
\frac{k_{1 p}(t)}{m \omega_{n}{ }^{2}}=\left\{\begin{array}{ccc}
F k_{1} /\left(m \omega_{n}{ }^{2}\right) & \text { if } & 0<t \leq \tau / 2 \\
k_{1} /\left(m \omega_{n}{ }^{2}\right) & \text { if } & \tau / 2<t \leq \tau
\end{array},\right.
$$

where $F$ is a factor characterizing the tooth engagement number. The pairs $F-1$ are given above the stability charts in Fig. 5. For example, 2/3-1 corresponds to the case, when the number of active teeth alternates between 2 and 3 . The case of $-1-1$ may look unrealistic, but we still presented it since this could be viewed as a kind of prediction for the case when the so-called backward milling is also involved (see similar effects described in Bayly et al., 1999).

The stability charts for varying spindle speed are presented in Fig. 6 and Fig. 7. The charts in Fig. 6 are constructed for the power series approximation (see Eq. (6)) of the varying time delay, while the charts in Fig. 7 are calculated for the exact model Eq. (4). The charts are categorized with the help of the time periodicity / average time delay ratio $\left(T / \tau_{0}\right)$, and the delay variation amplitude / average time delay ratio $\left(\tau_{1} / \tau_{0}\right)$, according to Eq. (22). All stability charts were computed for the approximation parameter $n=20$.

Figure 7 shows the same stability charts for the same categories as Fig. 6, but the stability domains are calculated by means of the semi-discretization method applied for the exact equation of motion Eq. (4) directly. The comparison of the two series of charts in Fig. 6 and 7 makes it possible to check the validity of the power series approximation for the varying delay as shown by Eq. (6).
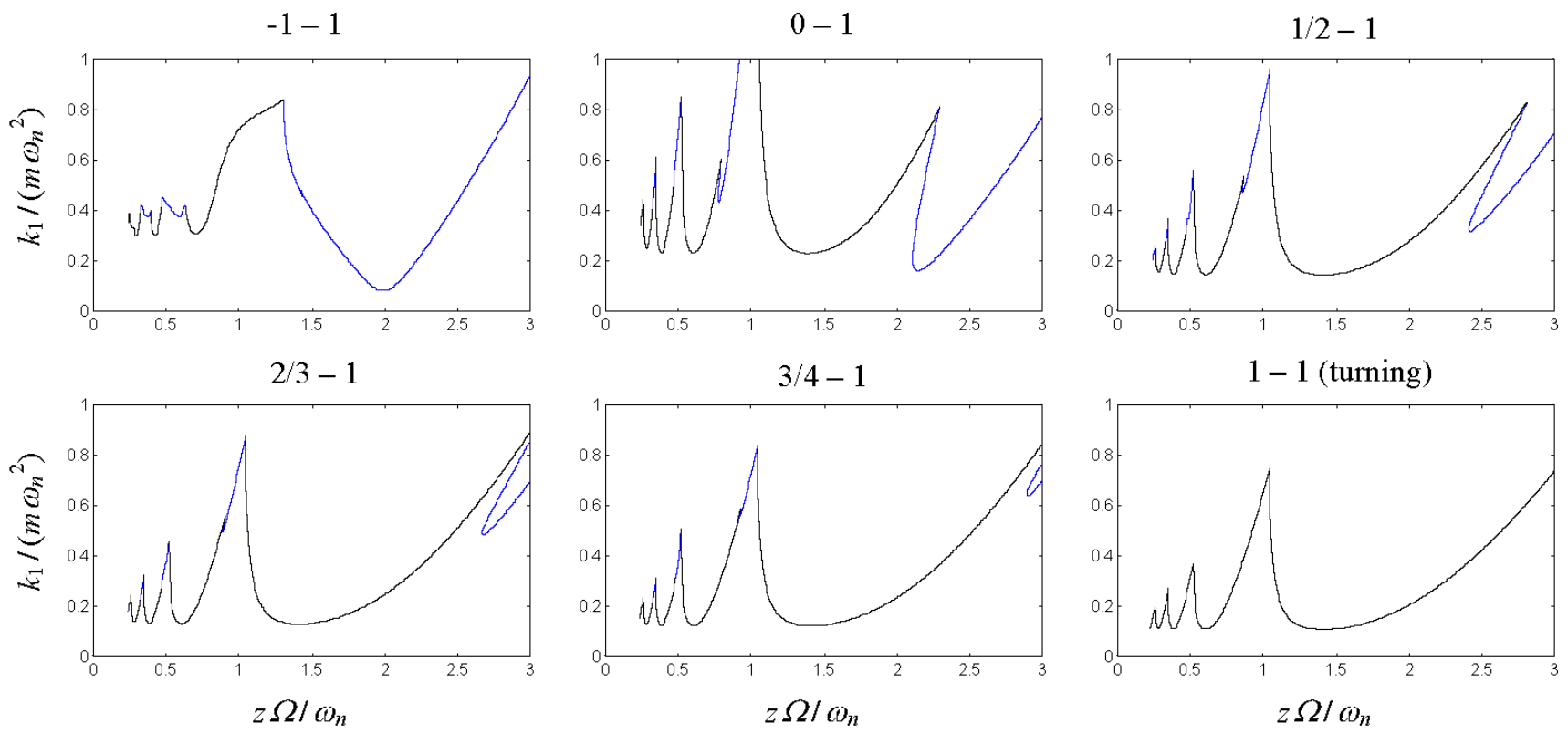

Fig. 5. Stability charts for milling process governed by Eq. (3) 

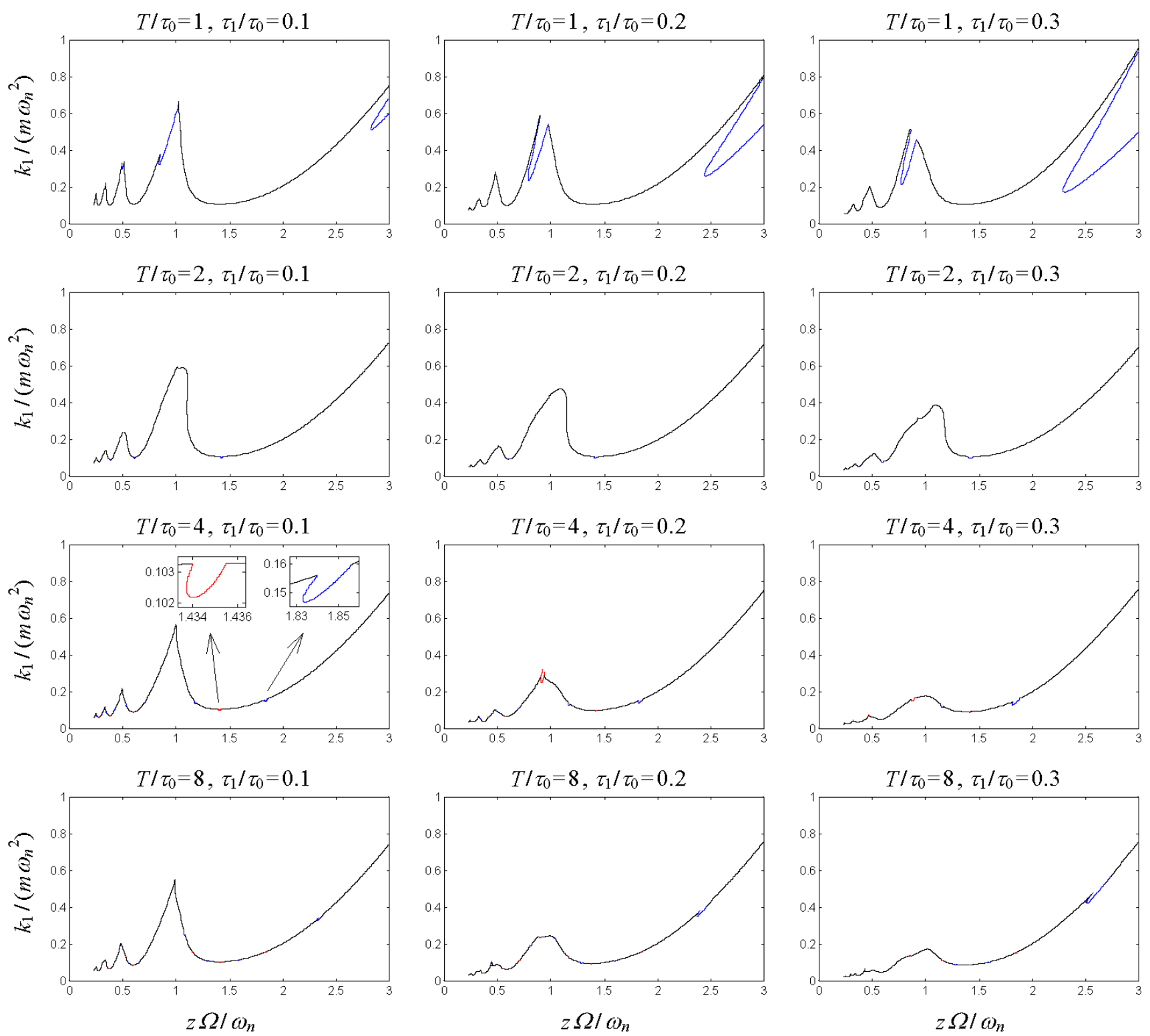

Fig. 6. Stability charts for turning process with varying spindle speed governed by the approximating Eq. (6)

In all the charts, the black stability boundary curves refer to Hopf bifurcations, that is, self-excited vibrations arise at the loss of linear stability. The spectrum of these vibrations contain two frequencies: one is the frequency $2 \pi / T$ of the parametric excitation, while the other is related to the angle of the characteristic root crossing the unit circle. The blue stability boundary curves refer to flip, or period doubling bifurcations, that is, the occurring self-excited vibrations have time period $2 \mathrm{~T}$ at the stability limit.

In Fig. 6 and 7, red boundary curves also show up. These refer to bifurcations topologically equivalent to the saddle-node bifurcations of autonomous systems. These new kinds of vibrations have never been identified in machine tool vibrations. It is likely, that two independent T-periodic motions will arise at these stability limits, but their existence, stability analysis, etc., should be the goal of further investigation in this direction. Experimental identification will also be difficult due to the narrow parameter range where these bifurcations arise.

The existence of these new kinds of vibrations with varying spindle speed is not surprising since in the section on "Models of time varying cutting", the possible existence of all the three kinds of bifurcations were shown analytically.

Note that the Hopf bifurcation stability limits are often fragmented by small boundaries of period doubling and/or saddle-node bifurcations (see enlarged parts of Fig. 6 and 7). 

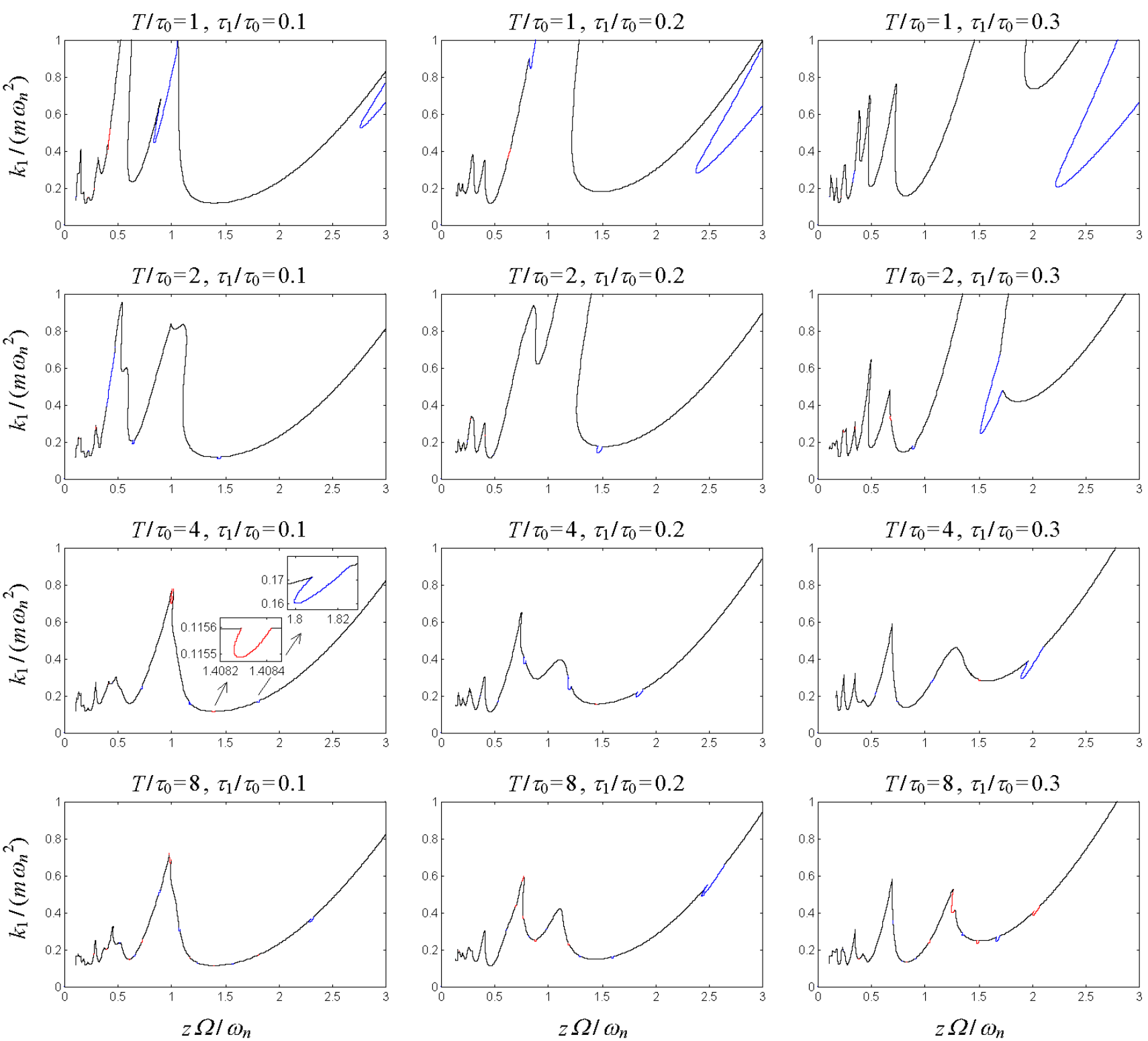

Fig. 7. Stability charts for turning process with varying spindle speed exactly described by Eq. (4)

\section{CONCLUSIONS}

The analytical, numerical and experimental stability analysis of cutting processes in case of high-speed and/or low immersion milling have presented new kinds of instability lobes related to period doubling bifurcations in the dynamics of machine tools. In the present pilot study, these new observations of the specialist literature were compared to the dynamics of cutting with varying cutting speed.

When the time periodicity in the cutting speed is equal to the time delay in the system, similar instability lobes show up in the stability charts at high values of average cutting speeds as in case of the milling process. This phenomenon was suspected form the slight similarities of the corresponding mathematical models. In spite of the fact, that in the model of milling a coefficient is time-periodic, and in the model of timevarying cutting the time delay itself is time-periodic, a Taylor series approximation with respect to the delay variation amplitude in the second case explores similarity between the two models.

This type of approximation, however, can be used cautiously, since the higher order expansion of the delay variation term would result higher derivatives of delayed terms in Eq. (5). The DDE, where the highest derivative is delayed, is called advanced differential equation, and, since the present value of the variable depends on the future state, it is always unstable. This contradiction can be explained if the weakness 
of the Taylor expansion is understood: it does not converge uniformly to the weight function of past states above the time in the past back to minus infinity. This also explains the relatively great quantitative differences between the exact and approximate stability charts of Fig. 6 and 7.

When the time periodicity is greater than the time delay (which is the realistic case in practice), the stability charts show even more complex, almost fractal-like structures. Apart of the period doubling bifurcations, saddle-node bifurcations of periodic orbits also occur. In the meantime, great improvements in stability properties can be expected at certain narrow parameter domains. The further study of the practical importance of these domains is an important further goal of this research work.

A new method was developed and applied to investigate the stability of the corresponding mathematical models, the time-periodic DDEs. The basic idea of the semi-discretization method is to use a finite dimensional approximation with respect to the past effect only, and investigate the stability of a high dimensional ODE instead of the infinite dimensional DDE. A 20 dimensional approximation is already good enough to identify 4-5 lobes quantitatively correctly in the stability charts.

\section{ACKNOWLEDGMENTS}

This research was supported by the Hungarian National Science Foundation under grant no. OTKA T030762/99, and the European Union COST P4 project.

\section{REFERENCES}

Balachandran, B., Zhao, M. X., 2000, A Mechanics Based Model for Study of Dynamics of Milling Operations, Meccanica, accepted.

Bayly, P. V., Young, K. A., Halley, J. E., 1999 Tool oscillation and the formulation of lobed holes in a quasi-static model of reaming, in Proceedings of the $17^{\text {th }}$ ASME Biennial Conferences on Vibration and Noise, ASME Design Engineering Technical Conferences, Las Vegas, Nevada, paper no. DETC99/VIB-8061 (CD-ROM).

Budak, E., Altintas Y., 1995, Analytical Prediction of Stability Lobes in Milling, Annals of the CIRP, 44, pp. 357-362.

Corpus, W. T., Endres, W. J., 2000, A High-Order Solution for the Added Stability Lobes in Intermittent Machining,
Proceedings of the Symposium on Machining Processes, Orlando, Florida, MED-11, pp. 871-878.

Davies, M. A., Pratt, J. R., Dutterer, B., Burns, T. J., 2000, Interrupted Machiening - A Doubling in the Number of Stability Lobes?, The Journal of Manufacturing Science and Engineering, in press.

Fargue, D., 1973, Réducibilité des systémes héréditaires á des systémes dinamiques, C. R. Acad. Sci. Paris 277B pp. 471473.

Farkas, M., 1994, Periodic Motions, Springer-Verlag, New York.

Hale, J. K., Lunel, S. M. V., 1993, Introduction to Functional Differential Equations, Springer-Verlag, New York.

Insperger T., Horváth R., 2000, Pendulum with Harmonic Variation of the Suspension Point, Periodica Polytechnica, 44, pp. 39-46.

Insperger T., Stépán G., 2000, Stability of the Milling Process, Periodica Polytechnica, 44, pp. 47-58.

Insperger T., Stépán, G., 2001, Semi-discretization of delayed dynamical systems, in Proceedings of ASME 2001 Design Engineering Technical Conferences, Pittsburgh, Pennsylvania, paper no. DETC2001/VIB-21446 (CDROM).

Minis, I., Yanushevsky, R., 1993, A new theoretical approach for the prediction of machine tool chatter in milling, Journal of Engineering Industry, 115, pp. 1-8.

Seagalman, D. J., Butcher E. A., 2000, Suppression of Regenerative Chatter via Impedance Modulation, Journal of Vibration and Control, 6, pp. 243-256.

Sexton, J. S., Stone, B. J., 1978, The stability of Machining with Continuously Varying Spindle Speed, Annals of the CIRP, 27, pp. 321-326.

Stépán, G., 1989, Retarded dynamical systems, Longman, Harlow.

Stépán, G., 1998, Delay-differential Equation Models for Machine Tool Chatter, in Dynamics and Chaos in Manufacturing Processes, Ed.: Moon, F.C., Wiley, New York, pp. 165-192.

Tlusty, J., Polacek, A., Danek, C., Spacek, J., 1962, Selbsterregte Schwingungen an Werkzeugmaschinen, VEB Verlag Technik, Berlin.

Tobias, S. A., 1965, Machine Tool Vibration, Blackie, London. 